[0212-7199 (2005) 22: 2: pp 88-90] ANALES DE MEDICINA INTERNA Copyright (C) 2005 ARAN EDICIONES, S.L.

AN. MED. INTERNA (Madrid) Vol. 22, N. ${ }^{\circ} 2$, pp. 88-90, 2005

\section{Trastorno doloroso y vértigo referido al oído. Derivación frecuente al ORL}

\author{
A. M. GARCÍA DE HOMBRE
}

Servicio de Otorrinolaringología. Hospital General de Fuerteventura
PAINFUL DISORDER AND VERTIGO REFERRED TO THE EAR. FRECUENT DERIVATION TO THE ENT SPECIALIST

\section{RESUMEN}

Hacemos una revisión sobre la disfunción témporomandibular o síndrome doloroso miofacial, entidad clínica que presenta una alta prevalencia en las consultas de otorrinolaringología (ORL). Se trata de pacientes polisintomáticos valorados en ORL procedentes de la consulta externa de Medicina Interna y de los médicos de cabecera. Como sintomatología acusan sensación vertiginosa, acúfenos y/o hipoacusia. Se discute el concepto, etiopatogenia, diagnóstico y tratamiento de este síndrome. Para el ORL es un desafío el escoger el tipo de tratamiento más adecuado con el fin de obtener un resultado alentador. A pesar de las múltiples publicaciones referidas a este tema siguen existiendo controversias en cuanto a la orientación de los enfermo y al tipo tratamiento.

PALABRAS CLAVE: Disfunción temporomandibular. Articulación temporomandibular. Dolor miofacial.

\begin{abstract}
We revise the temporomandibular dysfunction or miofacial painful syndrome, a clinical entity that shows a high prevalence at the ear, nose and throat (ENT) specialist's surgery. They are polisymtomatic patients seen at the ENT, which are sent by the Internal Medicine and family doctors. As symptoms the patients present vertiginous feeling, tinnitus and/or deafness. We discuss the concept, the ethiopathogenia, diagnosis and the treatment of this symdrome. For the ENT specialist it is a challenge to choose the most appropriate type of treatment in order to obtain a satisfying result. In spite of the great amount of issues referring to this subject, there are still controversies regarding the orientation of the patients and the type of treatment to use.
\end{abstract}

KEY WORDS: Temporomandibular dysfunction. Temporomandibular joint. Miofacial pain.

García de Hombre AM. Trastorno doloroso y vértigo referido al oído. Derivación frecuente al ORL. An Med Interna (Madrid) 2005; 22: 88-90.

\section{INTRODUCCIÓN}

Desde hace varios años y últimamente de manera cada vez mayor apreciamos que aumentan las interconsultas que nos solicita el Servicio de Medicina Interna y los médicos de cabecera de enfermos que aquejan dolor recurrente de oídos y sensación vertiginosa y a la exploración se constata el conducto auditivo externo y la membrana timpánica normal.

En la mayoría de las veces se trata de un síndrome doloroso de la articulación temporomandibular (ATM) que como síntoma principal tiene la otalgia y el vértigo. Muchas veces no se le dedica la suficiente atención en especial en atención primaria e indistintamente se derivan los pacientes al otorrinolaringólogo (ORL), dentista, máxilofacial y neurólogo. El ORL James Costen hace mención por primera vez de este síndrome en 1934 describiendo que reúne varios síntomas y signos que denotan la disfunción de la ATM $(1,2)$.
La disfunción dolorosa de la ATM o síndrome doloroso miofacial como se le denomina en la actualidad, fue descrito inicialmente como una neuralgia, caracterizado por otalgia, tinnitus, ocasionalmente vértigos, sordera catarral, cefalea e hipersensibilidad de la ATM $(3,4)$.

Este síndrome tiene una alta prevalencia y muchas veces es infravalorado y subdiagnosticado. En la inmensa mayoría se asocia a bruxismo nocturno y/o diurno. Se presenta tanto en niños como en adultos. Su prevalencia es mayor en mujeres entre 25-65 años con empeoramiento en período pre menstrual $(5,6)$.

En 1950, Moulten y cols. fueron fuertes propulsores de la idea de que los rasgos de la personalidad y otros factores psicológicos contribuían a la génesis de la disfunción de la ATM. En sus trabajos de psicoanálisis, describió cuatro aspectos distintos de factores psicológicos asociados con la disfunción de la $\operatorname{ATM}(11)$ :

Trabajo aceptado: 1 de diciembre de 2004

Correspondencia: Alina María García de Hombre. Servicio de Otorrinolaringología. Hospital General de Fuerteventura. Ctra. del Aeropuerto, km. 1. 35600 Puerto del Rosario. Fuerteventura. e-mail: aliadal@terra.es 
- Los pacientes con dolor de la ATM suelen caracterizarse como individuos dependientes que han sufrido la pérdida de soporte individual, individuos obsesivos, perfeccionistas, que tienen trabajo de mucha presión $(7,8)$.

\section{CARACTERÍSTICAS ANATÓMICAS}

La ATM está formada por el cóndilo de la mandíbula y la fosa glenoidea de la escama del hueso temporal. Es una gínglimodiartrosis que combina movimientos de rotación y desplazamiento. Externamente la rodea la cápsula articular, en su interior está revestida por una membrana sinovial laxa que produce un líquido mucinoso, que permite la nutrición de los elementos avasculares y la lubricación articular. Tiene un disco articular compuesto por tejido fibroso, que está fuertemente adherido al cóndilo mandibular por un lado y a la eminencia articular convexa por el otro (en el extremo anterior de la articulación) determinando la existencia de dos espacios articulares independientes entre sí, uno superior y otro inferior, de gran importancia funcional.

La irrigación depende de las arterias timpánica y auricular ramas de la arteria maxilar y por la arteria temporal superficial. Una rica red venosa del plexo pseudocavernoso, se ubica en la zona posterior. La inervación está a cargo del nervio aurículotemporal en la zona posterior y por ramas de los nervios de los músculos pterigoideo lateral, masetero y temporal (9).

\section{FISIOLOGÍA}

La ATM es la articulación que más se usa en el hombre. Constantemente se opone a la fuerza de gravedad, constituye una unidad funcional con su homónima contralateral. Participa activamente en la dentición, la oclusión dentaria, la masticación, la deglución, la respiración y la fonación. Sólo el reflejo de la deglución se realiza 2.000 veces por día. Por su ubicación en el macizo facial participa en la expresión emocional ya sea de tensiones o de relajación. Esta particularidad hace que los factores emocionales actúen como gatillantes del dolor, lo que ha sido demostrado por varios autores en diversas series de pacientes.

La parte superior de la articulación tiene solo un movimiento de deslizamiento ánteroposterior. La porción inferior tiene mayor movilidad, permite movimientos del cóndilo giratorios y en bisagra, lo cual es de gran utilidad en la masticación favoreciendo su desplazamiento lateral en hasta $2 \mathrm{~mm}$, hacia el lado que está trabajando. La proximidad articular hace que exista una gran variedad de síntomas óticos, como hipoacusia, vértigo, acúfenos y dolor. En especial los síntomas de vértigo y acúfeno son los que causan mayor disconfort. Muchas veces son pacientes en los se asocia hipertensión arterial, diabetes mellitus, dislipemia, anemia u otras entidades clínicas que per sé pueden cursar con tinnitus y los enfermos no mejoran a pesar de estar controlados. En realidad es la disfunción de la ATM la responsable de la persistencia de la sintomatología.

Es sorprendente la morbilidad que puede ocasionar el mal funcionamiento de esta articulación. Los síntomas pueden ser tan diversos como cefalalgia crónica, sensación de oído ocupado, hipoacusia, percepción de estar mareado o inestable $(10,11)$.
La bipedestación en el hombre ha provocado adaptaciones tanto morfológicos como funcionales en la ATM.

La disfunción temporomandibular es un trastorno sindrómico de la articulación temporomandibular, la musculatura y estructuras asociadas que la rodean, caracterizado por síntomas de dolor muy variados, limitación o desviación en la movilidad articular, ruidos articulares ocasionales y hábitos bruxitas, acompañados frecuentemente de una relevante sintomatología psicopatológica $(1,3)$.

Dentro de las causas que ocasionan este síndrome destacan las anormalidades intrínsecas que incluyen aberraciones del desarrollo como aplasias, hipoplasias e hiperplasias de una o más estructuras. También la articulación puede estar afecta por traumatismos, infección, inflamación o neoplasias $(7,12$ 14).

En general los factores psicológicos influencian, directa o indirectamente, en la etiología, perpetuación y tratamiento de prácticamente la totalidad de los casos de disfunción de la ATM. Su reconocimiento temprano evita fracasos en la terapéutica médica.

El diagnóstico y manejo del vértigo, la otalgia y el acúfeno puede ser muy simple y a la vez muy complejo y frustrante para el médico y para el paciente. El conocimiento de la neuroanatomía en especial el origen, trayecto y distribución de los pares craneales es esencial.

Se cuenta con varios recursos para el estudio de este trastorno como la palpación articular, auscultación, ecografía, artrografía, radiografía simple, electromiografía, artroscopia y la resonancia magnética. Esta última ha revolucionada el estudio de esta articulación llegando a una precisión diagnóstica del $90 \%$. La exploración vestibular y audiológica resulta normal (15-17).

\section{TRATAMIENTO}

En cuanto a las opciones terapéuticas lo más importante en estos casos es escuchar al paciente y saber canalizar su problema, con el fin de eliminar un dolor que se ha prolongado por mucho tiempo. Darle psicoterapia para lograr una armonización psicofuncional es fundamental. Los pilares del tratamiento son:

\section{CALMAR EL DOLOR}

-El uso de antinflamatorios no esteroideos (AINE), si bien no cambian el curso de la enfermedad controlan la inflamación aguda y mejora los síntomas. El uso de narcóticos no ofrece ventajas respecto a los AINE (18).

-Bloque articular con anestésico local (silocaína 2\% o bupivacaína)

\section{RELAJAR LA MUSCULATURA}

- Acostándose temprano, tomando un relajante muscular, comer dieta blanda y no consumir psicoestimulantes nocturnos, como el café (19).

\section{REHABILITACIÓN ARTICULAR CUANDO HAY DOLOR Y RESALTE ARTICULAR}

- Abrir ampliamente la boca y cerrarla 10 veces por día. 
- Reproducir este ejercicio, aplicando resistencia con el puño bajo el mentón.

\section{REHABILITACIÓN ARTICULAR CUANDO HAY DOLOR, RESALTE ARTICULAR Y LATERALIZACIÓN}

- Con la boca cerrada poner la punta de la lengua en posición retrodental apoyándola en el paladar, luego llevarla lo más atrás posible sin perder el contacto con el paladar y simultáneamente ir abriendo la boca. Mantener esta posición al menos 10 segundos. Descansar y repetir.

Estos ejercicios deben de repetirse varias veces al día de preferencia antes de las comidas.

\section{MEJORÍA DE LA MORDIDA}

- Se recomienda por parte del ortodoncista reposición de las piezas dentarias deficitarias, de preferencia las posteriores.

- Plano de relajación nocturno con dispositivo intraoral para relajar los músculos de la masticación, corregir el bruxismo y redistribuir el sentido de las fuerzas entre los dientes, los músculos y la ATM.

Sobre la ortodoncia comentan los autores desde su papel como placebo hasta efecto analgésico solo cuando hay sinovitis articular (20).

\section{Bibliografía}

1. Wazen JJ. Referred otalgia. Otolaryngol Clin North Am 1989; 22: 1205-15

2. Mix H. Síndrome doloroso de la articulación temporomandibular. Rev Otorrinolaringol Cir Cuello 1996; 56: 143-48.

3. Bush FM, Harkins SW, Harrington WG. Otalgia and aversive symptoms in temporomandibular disorders. Ann Otol Rhinol Laryngol 1999; 108: 884-92.

4. Ciancaglini R, Loreti P, Radaelli G. Ear, nose, and throat symptoms in patients with TMD: the association of symptoms according to severity of arthropathy. J Orofac Pain 1994; 8: 293-7.

5. Blake P, Thorburn DN, Stewart IA. Temporomandibular joint dysfunction in children presenting as otalgia. Clin Otolaryngol 1982; 7: 237-44.

6. Bonjardim LR, Gaviao MB, Carmagnani FG, Pereira LJ, Castelo PM. Signs and symptoms of temporomandibular joint dysfunction in children with primary dentition. Clin Pediatr Dent 2003; 28: 53-8.

7. Munera MM, Sierra CH, Jiménez JD. Disfunción de la articulación Temporo Mandibular en pacientes con prognatismo mandibular con y sin tratamiento ortodóncico-quirúrgico. CES Odontol 1995; 5: 113-8.

8. González de River JL, De la Hoz JL, Rodríguez-Abaín MJ, Monterrey AL. Perfil psicopatológico en pacientes con disfunción temporomandibular. An Psiquiatr 1999; 3: 91-5.

9. Incesu L, Taskaya-Yilmaz N, Ogutcen-Toller M, Uzun E. Relationship of condylar position to disc position and morphology. Eur J Radiol 2004; 51: 269-73.

10. Tuz HH, Onder EM, Kisnisci RS. Prevalence of otologic complaints in patients with temporomandibular disorder. Am J Orthod Dentofacial Orthop 2003; 123: 620-3.

11. Kuttila SJ, Kuttila MH, Niemi PM, Le Bell YB, Alanen PJ, Suonpaa JT. Secundary otalgia in an adult population. Arch Otolaryngol Head Neck Surg 2001; 127: 401-5.

12. Schulte JK, Anderson GC, Hathaway KM, Will TE. Psychometric pro-

\section{TRATAMIENTO QUIRÚRGICO}

- Cirugía ortognática en trastornos oclusales y malformaciones morfológicas de la mandíbula.

- Artroplastia solo en caso de anquílosis severa o tumor articular.

A pesar de lo mucho que se ha escrito sobre la repercusión funcional de los trastornos de la ATM sigue existiendo disparidad de criterios y controversias en cuanto al manejo y resultados terapéuticos.

Más recientemente se publican pacientes tratados con toxina botulínica y radiofrecuencia $(21,22)$.

En lo ante expuesto hemos tratado de sintetizar todos las posibilidades terapéuticas que se mencionan en la literatura. Siempre cuando hay muchas alternativas para tratar una enfermedad nos dice que no existe una que sea totalmente efectiva.

\section{CONCLUSIONES}

Los médicos que realizan atención primaria pueden proporcionar un servicio valioso al descartar otras enfermedades y diagnosticar inicialmente la disfunción por dolor miofacial. Si bien no tienen a cargo el tratamiento del paciente pueden cooperar a que se realice una derivación adecuada para el diagnóstico y tratamiento definitivo, evitando con ello el paso del enfermo por Medicina Interna o Neurología. files and related pain characteristics of temporomandibular disorder patients. J Orofacial Pain 1993; 7: 247-53.

13. Buranastidporn B, Hisano M, Soma K. Articular disc displacement in mandibular asymmetry patients. J Med Dent Sci 2004; 51: 75-81.

14. Bauss O, Sadat-Khonsari R, Fenske C, Engelke W, Schwestka-Polly R. Temporomandibular joint dysfunction in Marfan syndrome. Oral Surg Oral Med Oral Pathol Oral Radiol Endod 2004; 97 (5): 592-8.

15. Al-Ani MZ, Davies SJ, Sloan P, Rushton Br. Does the procedure of arthrography affect the range of movement in patients with locking of the temporomandibular joint? J Oral Maxillofac Surg 2004; 42: 335-6.

16. Brandlmaier I, Rudisch A, Bodner G, Bertram S, Emshoff R. VE, Horner K, Gray RJ. Temporomandibular joint internal derangement: detection with 12.5 MHz ultrasonography. J Oral Rehabil 2003; 30: 796-801.

17. Yura S, Totsuka Y, Yoshikawa T, Inoue N. Can arthrocentesis release intracapsular adhesions? Arthroscopic findings before and after irrigation under sufficient hydraulic pressure. J Oral Maxillofac Surg 2003; 61: 1253-6.

18. Ta LE, Dionne RA. Treatment of painful temporomandibular joints with a cyclooxygenase-2 inhibitor: a randomized placebo-controlled comparison of celecoxib to naproxen. Pain 2004; 111: 13-21.

19. Lindsten R, Magnusson T, Ogaards B, Larsson E. Effect of food consistency on temporomandibular joint morphology: an experimental study in pigs. J Orofac Pain 2004; 18: 56-61.

20. Dervis E. Changes in temporomandibular disorders after treatment with new complete dentures. J Oral Rehabil 2004; 31: 320-6.

21. Martínez-Pérez D, García Ruiz-Espiga P. Recurrent temporomandibular joint dislocation treated with botulinum toxin: report of 3 cases. J Oral Maxillofac Surg 2004; 62 (2): 244-6.

22. Al-Badawi EA, Mehta N, Forgione AG, Lobo SL, Zawawi KH. Efficacy of pulsed radio frequency energy therapy in temporomandibular joint pain and dysfunction. Cranio 2004; 22: 10-20. 